\title{
Student's Corner-1
}

\author{
$\begin{array}{ll}\text { P. Krishnam Raju } & \text { A. N. Patnaik } \\ & \end{array}$ \\ ${ }^{1}$ Department of Cardiology, Care Hospital, Care Op Center, \\ Hyderabad, Telangana, India \\ 2Department of Cardiology, Sun Shine Hospitals, Hyderabad, \\ Telangana, India
}

Ind J Car Dis Wom:2020;5:165-167

\section{Q) What Are the Differences between Dyspnea and Easy Fatigability?}

\section{Response by Prof. Patnaik}

Both "dyspnea" and "easy fatigability" are common cardiac symptoms which are confusing in nature, as they are often interchangeably used by clinicians and students. Each of them denotes a different mechanism, and have distinctive clinical implications in the evaluation of a cardiac patient.

Dyspnea (also called shortness of breath) is used to refer to uncomfortable awareness of one's own breathing. The patient can also describe it as the inability to take deep breath or chest tightness. It should not be confused with tachypnoea (rapid rate of breathing), hyperpnoea (excess act of breathing), or hyperventilation. Dyspnea majorly occurs due to pulmonary or cardiac causes. The mechanism of this clinical symptom is not clearly understood. The major mechanism involves abnormal pulmonary mechanics and increase in the overall work of breathing. The peripheral receptors (respiratory muscle spindles, receptors in pulmonary parenchyma and central airways, and chemoreceptors in carotid bodies) have a complex relation with the central nervous system (cortex and medulla) through the vagi and afferent neurons in the spinal cord. Any excess and inappropriate respiratory output in response to the stimulation of the peripheral receptors can give rise to sensation of dyspnea. Left-sided cardiac lesions and left ventricular failure that increase the left atrial (LA) pressure and the resultant raised pulmonary venous hypertension affects the pulmonary mechanics. Orthopnea and paroxysmal nocturnal dyspnea are two important subtypes of dyspnea.

Fatigue is a more persistent subjective feeling of lack of energy and motivation, malaise, lethargy, weariness, and diminished mental functioning (difficulty in concentration and memory), which impairs the quality of life. Patient may use descriptions like feeling run down, worn out, listless, and tired. It may be confused with drowsiness and loss of vitality associated with aging in the elderly. It is more difficult to assess and quantify. Many medical conditions such as anemia, hypothyroidism, convalescence from infections
Address for correspondence Maddury Jyotsna, MD, DM, FACC, FESC, FICC, Nizam Institute of Medical Sciences (NIMS), Punjagutta, Hyderabad, Telangana 500082, India (e-mail: janaswamyjyostna@gmail.com).

like typhoid, tuberculosis, dengue, chikungunya, influenza, cancer, celiac disease, chronic fatigue syndrome, sleep apnea, use of chemotherapy, $\beta$-blockers or diuretics, and cardiac conditions like right heart failure can present with fatigue. The ultimate mechanism probably involves altered skeletal muscle metabolism and efficiency with or without reduced blood flow into the skeletal muscles.

In a patient afflicted with chronic heart failure, both dyspnea and fatigue may be present. Dyspnea is more to do with the respiration, and fatigue is about the whole body muscle function. A few additional questions may be needed to bring out which symptom the patient is trying to convey.

\section{Response by Prof. Krishnam Raju}

Dyspnea and easy fatigability are two different symptoms and are vague by their very nature.

Dyspnea could be caused by five different forms of pathophysiology:

1. Pulmonary parenchymal and vascular disease including upper respiratory forms of pathology. There are problems of ventilation, perfusion, mixed physiology, and perfusion ventilation mismatch.

2. Low-cardiac output due to any cardiac diverse pathologies.

3. Decreased oxygen carrying capacity, for example, anemia, etc.

4. Respiratory neuromuscular paralysis of diverse etiologies.

5. Psychiatric disorders, for example, anxiety reaction, conversion reaction, and hyperventilation syndrome

Easy fatigability could be due to a wide range of etiologies but basically due to decreased nutrients and/or oxygen supply/muscular disorders or neuromuscular disorders.

The etiologies could be as follows:

a. Metabolic causes like anemia, iron deficiency, and hypovitaminosis.

b. Low-cardiac output.

c. Hypoxemia.

d. Neuromuscular disorders.

e. Myopathies. published online

June 2, 2020
DOI https://doi.org/

10.1055/s-0040-1713344.
C2020 Women in Cardiology and Related Sciences
License terms

(요 (1) $\Theta \circledast$ 
f. Endocrinopathies, for example, diabetes mellitus, hypothyroidism, hypoadrenalism, etc.

g. Dyselectrolytemia, for example, hyponatremia, hypokalemia, hypomagnesemia, etc.

h. Chronic fatigue syndrome.

i. Drug-induced, for example, statins, opioids, cocaine, etc.

Key points-both symptoms could coexist; it is important to be familiar with local, regional, and colloquial language expressions used by the patients, as they often tend to not be well-versed with the language we converse in; frequently, the attendants of the patients talk and not the patient.

Dyspnea is generally associated with rest or exercise, hypoxia, myocardial dysfunction, and pulmonary arterial or venous hypertension (PAH). Investigations advised to be conducted in order to determine the cause of dyspnea are as follows:

1. Check for rest/exercise Spo2/arterial blood gases.

2. Check for myocardial dysfunction at rest and on exercise. In select cases, it is appropriate to carry out exercise echo diastology.

3. Check for PAH at rest and on exercise.

4. Six-minute walk test could be safe, cheap, and useful.

5. Pulmonary function tests.

6. Hemogram.

7. Check metabolic, electrolyte, and endocrine parameters in appropriate patients.

8. Cardiopulmonary exercise testing.

Key points-all the above tests are not needed in all subjects. Use them appropriately and also remember that both symptoms may coexist. The most important point to remember is to conduct a good clinical evaluation because it will lead us to do appropriate laboratory testing in order to answer our specific clinical questions. If you have no relevant targeted questions, you will not get useful answers, and it will be bad economics.

\section{Q) Why Paroxysmal Nocturnal Dyspnea (PND) Does Not Occur Even Though There Is High-Pulmonary Capillary Wedge Pressure (PCWP) in Chronic Constrictive Pericarditis (CCP)?}

\section{Response by Prof. Patnaik}

The well-described mechanisms of PND include the following: i) increase in plasma volume due to reabsorption of edema fluid from tissues to circulation; ii) shift of edema fluid from dependent parts and splanchnic region into lungs, leading to increased pulmonary blood volume and PCWP; also, there is transudation of blood serum into interstitial spaces; iii) inadequate lymphatic drainage; iv) decreased lung compliance reduced lung reserve volumes; v) bronchospasm and increased airflow; vi) hypoxia-induced increased capillary permeability; vii) altered alveolar surfactant function; and viii) elevated diaphragm in supine position.
In constrictive pericarditis, the main pathophysiology involves loss of pericardial compliance with preserved LV and $\mathrm{RV}$ systolic function and normal lung function. The compensatory mechanism is elevation of ventricular end-diastolic pressures to maintain cardiac filling and output, which eventually leads to primary diastolic heart failure. The noncompliant pericardium ensures a fairly fixed combined volume of LV and $\mathrm{RV}$ and ventricular interdependence. Since these changes are chronic, the circulatory system develops adequate compensatory mechanisms such as well-functioning lymphatics. The raised pulmonary venous pressures are modest and the multiple other factors responsible for precipitation of PND, mentioned earlier, are not seen in CCP, explaining its rarity in cases of constrictive pericarditis.

\section{Response by Prof. Krishnam Raju}

In spite of increase in LA pressure, patients with CCP do not generally have paroxysmal nocturnal dyspnea. The reason being that LA pressure generally does not cross $25 \mathrm{~mm} \mathrm{Hg}$, as the RV output and stroke volume are also restricted; hence, lung congestion does not develop. This is true of the common variety of CCP, where the constriction is global and circumferential. However, a small number of them do develop PND, as the very high systemic venous pressure in superior vena cava (SVC) territory leads to obstruction of thoracic lymphatic duct and bronchial venous congestion which, in turn, leads to lung congestion.

The following variants of CCP may also lead to PND:

1. Regional constrictive pericarditis producing pulmonary venous obstruction.

2. Regional CCP leading to severe left $A V$ groove fibrosis and calcification, extending into mitral valve and resulting in mitral stenosis; although, this is a rare clinical scenario.

3. Severe LV dysfunction secondary to CCP myocardial scarring or any other comorbidities like CAD, which could be due to fibrocalcific entrapment of coronaries, particularly left anterior descending (LAD) and, sometimes, right coronary (RC) arteries.

\section{Q) Is Hills Sign in Chronic Aortic Regurgitation a Barometric Error? Please Provide Justification.}

\section{Response by Prof. Patnaik}

Hill's sign is said to be positive when systolic cuff-leg pressure is more than $20 \mathrm{~mm} \mathrm{Hg}$ higher than the systolic cuff-arm pressure using the same cuff. It is a well-described clinical sign of significant aortic run-off (chronic aortic regurgitation). The accepted mechanism is as follows: the lower limb vessels are more muscular than upper limb arteries, and they are more in direct continuation of aorta. The forward pressure wave is transmitted at higher speed into the lower limb vessels, gets reflected and summated with the incoming wave, giving an apparent increase in the lower blood pressure. In aortic regurgitation, the increased momentum of ejection leads to greater amplitude of pressure wave; subsequently, 
the summation effects are greater with resultant higher systolic pressure in the lower limb vessels.

However, in a small Canadian study, ${ }^{1}$ it had been observed that intra-arterial pressure measurements do not show this phenomenon, and clinical validity of the Hill's sign was questioned. It was attributed as an artifact of sphygnomanometric measurement of lower limb pressure without any physiological basis. In spite of this important observation, it continues to be a popular clinical sign to evaluate significance of AR along with other signs of aortic run-off.

\section{Response by Prof. Krishnam Raju}

Hill's sign is elicited by measuring systolic blood pressure in the arm and the leg simultaneously, or in very quick succession, using the regular adult cuff. Measurements are taken over brachial artery and popliteal or posterior tibial arteries. Intra-arterial invasive femoral artery is not acceptable, as it is not peripheral enough for this sign. Due to the muscular nature of the peripheral arteries, they allow rapid transmission of the pulse wave, resulting in the summation of the incident and reflected waves, and this effect is more in the legs than in the arm. Normally, the difference is 15 to $20 \mathrm{~mm}$ of $\mathrm{Hg}$. In other hyperdynamic circulatory conditions, this difference is not more than $40 \mathrm{~mm}$ of $\mathrm{Hg}$. The difference of more than 40 to $60 \mathrm{~mm}$ of $\mathrm{Hg}$ is indicative of aortic run-off conditions, typically aortic regurgitation and similar hemodynamic situations such as rupture sinus of Valsalva or large patent ductus arteriosus (PDA) with large shunt. The difference of more than $60 \mathrm{~mm}$ of $\mathrm{Hg}$ is suggestive of severe aortic regurgitation.

This pressure difference is due to multiple reasons:

1. The pressure reflecting sites are more in the legs than arms.

2. Leg arteries are more muscular than arm vessels.

3. Age-related arterial compliance changes are more in the legs than arms.

4. Upper arm vessels arise at a 90-degree angle from the arch; as a result of Venturi effect, central aortic and arm pressures are similar to leg pressures as they are remotely located

5. In patients of AR with Hill's sign is useful for follow-up to assess increasing regurgitation and also pick up LV dysfunction when pressure difference is progressively less.

6. In the current era of echocardiography, this sign has very little value, rarely ever used in real-world clinical practice, and has become more of curiosity value for hemodynamic understanding and discussions

\section{Conflict of Interest}

None declared.

\section{Reference}

1. Kutryk M, Fitchett D. Hill's sign in aortic regurgitation: enhanced pressure wave transmission or artefact? Can J Cardiol 1997; 13(3):237-240 